\title{
Effects of experimental acute myocardial infarction on blood cell counts and plasma biochemical values in a nude rat model (Crl:NIH-Fox1 ${ }^{\text {RNU }}$ )
}

\author{
Zuhair Bani Ismail • Mahmoud Abu Abeeleh • Khaled R. Alzaben • \\ Sami A. Abu-Halaweh • Abdel Karim S. Aloweidi • Iyad A. Al-Ammouri • \\ Mohamed K. Al-Essa • Samir K. Jabaiti • Moaath M. Alsmady • Ahmad Al-Majali
}

Published online: 26 March 2009

(C) Springer-Verlag London Limited 2009

\section{Erratum to: Comp Clin Pathol DOI 10.1007/s00580-009-0827-6}

There are two errors in the Materials and methods section:

1. In the single sentence in the subsection "AMI assessment", "serum" should be "plasma". The correct sentence is: "The occurrence of AMI in rats was assessed by measuring plasma concentration of $\mathrm{LDH}, \mathrm{CK}$, and CK-MB before surgery (T0), and at 6 and $24 \mathrm{~h}$ following AMI induction."

2. In the first paragraph of the subsection "Blood and plasma sample collection", the weight range of the animals studied was given incorrectly. The correct sentence is: "Whole blood was collected from 50, 5-to 7-week-old (250-350 g body weight), male nude rats, strain Crl:NIH-Fox1rnu between the months of October and December, 2007."

The online version of the original article can be found at http://dx.doi. org/10.1007/s00580-009-0827-6

Z. Bani Ismail $(\bowtie) \cdot$ A. Al-Majali

Department of Veterinary Clinical Sciences,

Faculty of Veterinary Medicine,

Jordan University of Science and Technology,

Irbid 22110, Jordan

e-mail: zuhair72@just.edu.jo

M. Abu Abeeleh

Department of Cardiothoracic Surgery,

Faculty of Medicine,

University of Jordan,

Amman, Jordan

K. R. Alzaben · S. A. Abu-Halaweh • A. K. S. Aloweidi

Department of Anesthesiology, Faculty of Medicine,

University of Jordan,

Amman, Jordan

\section{A. Al-Ammouri}

Department of Pediatrics, Faculty of Medicine,

University of Jordan,

Amman, Jordan

M. K. Al-Essa

Department of Physiology, Faculty of Medicine,

University of Jordan,

Amman, Jordan

S. K. Jabaiti • M. M. Alsmady

Department of Plastic Surgery, Faculty of Medicine,

University of Jordan,

Amman, Jordan

Present Address:

M. K. Al-Essa

Philadelphia Biological and Medical Product Development

Centre,

Amman, Jordan 